\title{
Acuerdos de promoción comercial y contrataciones estatales: especial referencia a los límites de los poderes públicos en el espacio jurídico global Commercial marketing agreements and state contracts
}

\author{
VERÓNICA ROJAS MONTES*
}

Resumen: El artículo analiza la relación entre el régimen general de las contrataciones estatales y el Acuerdo de Promoción Comercial suscrito entre el Perú y los Estados Unidos de América, en el contexto de la globalización jurídica. A partir de este caso, destaca cómo se limitan los poderes públicos nacionales en el espacio jurídico global, y cómo la autodeterminación normativa viene a ser relativizada porque prevalece el ordenamiento jurídico global al nacional. Esto significa que la globalización jurídica cambia el rol del Estado y relativiza su soberanía.

Palabras clave: Globalización jurídica - soberanía - Estado - contrataciones estatales - Acuerdo de Promoción Comercial - licitación pública - contratación directa

Abstract: This article analyzes the relation between the Peruvian legal framework applicable to government procurement and the United States - Peru Trade Promotion Agreement in a context of legal globalization. This article emphasizes that the decisions of domestic administrators are increasingly constrained by regulations established on the global level, limiting the decision making process of local authorities. This means that in a legal globalized world the role of the Government and its sovereignty have changed.

Key words: Global administrative law - sovereignty - state - government recruitments - Trade Promotion Agreement - open tendering procedure limited tendering

CONTENIDO: I. EL ESPACIO JURÍDICO GLOBAL COMO CONTEXTO NECESARIO.- II. CONTRATACIONES ESTATALES PERUANAS Y EL TLC PERÚESTADOS UNIDOS DE AMÉRICA.- III. REFLEXIÓN FINAL.

\footnotetext{
* Es profesora de Derecho Administrativo 2 en la Pontificia Universidad Católica del Perú. A la vez, posee el grado de magister artis en Administración Pública otorgado por el Instituto Universitario Ortega y Gasset, adscrito a la Universidad Complutense de Madrid en convenio con el Instituto Nacional de Administración Pública de España. Asimismo, es doctoranda en Derecho por la Pontificia Universidad Católica del Perú y abogada asociada senior del estudio Muñiz, Ramírez, Pérez-Taiman \& Olaya Abogados.
} 


\section{EL ESPACIO JURÍDICO GLOBAL COMO CONTEXTO NECESARIO}

Los conceptos de "globalización" o "mundialización" surgieron en los dos últimos decenios después de la desaparición de la Unión de Repúblicas Socialistas Soviéticas y han sido utilizados desde su inicio, generalmente con referencia a la creación de un mercado global, de bienes y de capital financiero, con pocas fronteras arancelarias, así como también a los procesos de comunicación entre las sociedades mundiales que se han acelerado de manera extraordinaria debido a los adelantos técnicos (computadoras, internet, satélites que facilitan la comunicación telefónica y de imágenes, etcétera).

Los conceptos mencionados se refieren a procesos reales tanto en el ámbito productivo, como son los flujos de capitales o el tránsito de personas, así como en todo tipo de procesos vinculados a la comunicación virtual y real entre los diversos actores. Existe, pues, como menciona el profesor Cassese al hacer referencia a la interdependencia de los países en el ámbito internacional en épocas de la globalización - y en diversos aspectos-, una conexión inexorable con el espacio jurídico.

Específicamente, Cassese nos dice que la interdependencia del conjunto de los países del mundo es uno de los aspectos de la globalización que ha sido producida por factores como los avances de las tecnologías de la comunicación, el aumento de las transacciones internacionales y de su volumen, así como por los flujos internacionales de capitales ${ }^{1}$. En palabras de Ortega, "se debe destacar la presencia de cuatro tipos de actores que interactúan en el espacio jurídico mundial: los Estados, las organizaciones internacionales de poderes públicos, las empresas multinacionales y las asociaciones privadas de nivel internacional, lo que nos proporciona la considerable cifra de 1.850 actores"2.

Los espacios en los que influye indiscutiblemente la globalización jurídica son los de comercio internacional, derechos de propiedad intelectual, derechos humanos, medio ambiente, entre otros.

En efecto, el presente trabajo analizará los tipos de entidades globales, sus características y las normas que del APC se derivan como vinculantes para nuestro Estado, con énfasis en el problema sobre las contrataciones públicas de nuestro país y su ubicación en el contexto de la globalización jurídica a través del acuerdo de promoción comercial (Tratado de Libre Comercio, en adelante TLC) ${ }^{3}$ suscrito con los Estados Unidos de América.

1 Cassese, Sabino. La globalización jurídica. Primera edición. Madrid: Marcial Pons, 2006, p. 13.

2 lbíd., p. 9.

3 Si bien el nombre legal es Acuerdo de Promoción Comercial firmado entre la República del Perú y los Estados Unidos de América, nos referiremos a él como TLC (Tratado de Libre Comercio) a lo largo de la investigación, de la misma manera en que se lo denominó desde un inicio de las negociaciones. 


\section{I.1. Tipos de entidades globales}

Ciertamente, la realidad global es compleja por varias razones, pero, principalmente, por tres. En primer lugar, existen más de 1800 organizaciones internacionales (de Estados, organizaciones no gubernamentales, empresas, bancos, etcétera), lo que hace prolífica la producción normativa o los estándares emanados de estas entidades. En segunda instancia, la ausencia de un solo centro de poder mundial en función al cual graviten las organizaciones internacionales —se dice que el ámbito global es headless, además de policéntrico y que cada centro de poder es "soberano"-.

Cassese, dentro de un reciente trabajo sobre casos, materiales y temas del Derecho administrativo global, editado con la participación de la New York University School of Law y el Istituto di Ricerche sulla Pubblica Amministrazione, afirma que el ordenamiento legal global posee un denso cúmulo de organizaciones que pueden estructurarse a grandes rasgos en dos categorías:

The first is composed of "horizontal" transgovernmental bodies, which have three main features. To begin with, these committees are autonomous or "headless", as they are not incorporated into an international organization $(\mathrm{IO})$.

In addition, they are not regulated by treaties, but rather by informal agreements between independent or quasi-independent national agencies. They do not make formal decisions, binding upon States. Finally, they exist for different reasons - for example, to coordinate or facilitate information-sharing among national regulators, to draft guidelines and spread best practices, or to set (legally nonbinding) international standards. The most well-known examples of such bodies are the International Organization of Securities Commissions (IOSCO), the International Association of Insurance Supervisors (IAIS), the G-10 committees, such as the Committee on the Global Financial System, the Committee on Payment and Settlement Systems, and the Basel Committee on Banking Supervision (hereafter, Basel Committee; see supra Ch. 1.5).

The other category comprises "vertical" transgovernmental bodies. These are, by contrast, auxiliary or secondary bodies operating within IOs to set harmonization or standardization rules or to monitor the correct implementation of decisions. These bodies are typically composed not only of national (middle or high-level) officials but also of supranational officials, i.e. civil servants working within international secretariats. As a consequence, these fora open up national systems not only "laterally", to promote dialogue between domestic administrations, 
but also "vertically", to foster cooperation among supranacional and national bodies. This group of "mixed" or "vertical" transgovernmental networks includes most of the EU committees (comitology or executive committees, Council or legislative committees, and expert governmental committees, assisting the Commission), and most IO auxiliary bodies (to name but a few, WTO secondary bodies administering multilateral or plurilateral agreements; the UN "functional committees", exercising consultative functions; and the so-called Codex Committees, assisting the Codex Alimentarius Commission in the drafting of food-safety standards) $)^{4}$.

Otros autores sostienen que hay hasta cinco tipos de entidades internacionales, pero bastan estas dos clases de entidades transgubernamentales "verticales" y "horizontales" como categorías gruesas, ya que se encargan de agrupar la amplia gama de organizaciones. Entre las entidades transgubernamentales "verticales" tenemos a la Unión Europea como organización supranacional y a la Organización Mundial del Comercio en su calidad de organismo internacional. Para el caso de las "horizontales", que no están integradas por Estados, sino por diversas entidades privadas, podemos ubicar entre ellas a la International Association of Classification Societies LTD., la cual establece reglas y estándares técnicos comunes dedicados a asegurar que los bancos sean seguros, o el Basel Committee on Banking Supervision, organización que agrupa a los bancos de los trece países más desarrollados y poderosos económicamente, aquellos que establecen reglas comunes para el funcionamiento de los bancos en todo el mundo.

En tercer término, la enorme gama de temas tratados globalmente -ambiente, derechos de propiedad intelectual, comercio, banca, seguridad marítima, salud, alimentación, biodiversidad, telecomunicaciones, calidad, entre otros- genera una prolifica normativa global que se combina en los múltiples espacios de regulación internacional; ello ocasiona que esta normativa global se complemente, superponga o contradiga, de modo que se terminen por generar conflictos que se solucionan en el seno de cada organización internacional, cuando en realidad estos podrían llegar a resolverse de manera distinta.

Un caso ilustrativo de conflicto entre las normas domésticas y las globales es el estudiado por nosotros, en donde se yuxtaponen regulaciones vinculantes y no vinculantes sobre las contrataciones estatales en el acuerdo bilateral del TLC entre Perú y Estados Unidos de América.

4 Cassese, Sabino et al. Global administrative law. Cases, materials, issues. Segunda edición. Istituto di Ricerche sulla Pubblica Amministrazione e Institute for International, Law and Justice of the New York University School of Law, 2008. <http://www.iilj.org/GAL/documents/GALCasebook2008.pdf>. 
A partir de ello surgen interrogantes que ponen en la palestra la polémica premisa que cualquier asunto interno debe situarse y adecuarse al escenario de la globalización jurídica: icómo se entiende la soberanía de los Estados en la globalización jurídica?, iqué normativa se aplicará en el ámbito doméstico cuando existe conflicto de normas a nivel global en diversas materias?

Claramente, se observa que un conjunto completo de normas provenientes de diversas organizaciones generales, internacionales y sectoriales a nivel mundial — por ejemplo, organismos internacionales, organismos supranacionales u organizaciones transnacionales, numerosas, fragmentadas y sin un solo centro de poder- presentan cuatro características:

1. normas imperativas o indicativas;

2. producen muchos efectos "laterales" por su extraterritorialidad;

3. ausencia de una rígida división de tareas - la UE, la OMC, la OMPI, la FAO y la CBD pueden emitir normas sobre lo mismo-;

4. tienen "origen diverso, internacional, supranacional y nacional, por lo que puede decirse que el Derecho global no coincide con el Derecho internacional: este último sería una parte del primero"s.

\subsection{La soberanía relativa de los Estados nacionales en el espacio jurídico global}

En efecto, algo de lo que se está ocupando el Derecho administrativo es el estudio de la relativización de la soberanía de los Estados modernos (post revolución francesa), dentro de este marco jurídico global, pues en él se ubica una serie de normas que determinan el contenido de los ordenamientos jurídicos domésticos.

En el caso particular del Perú, en el año 2008 el Poder Ejecutivo promulgó 99 decretos legislativos para adecuar la normativa peruana al Tratado de Libre Comercio suscrito entre el Perú y los Estados Unidos de América, que entró en vigencia el primero de febrero de 2009. Esto es una muestra de lo que ya viene ocurriendo en el mundo hace décadas: los países soberanos deben adecuarse, necesariamente, a las regulaciones globales de los diversos foros internacionales. Los ordenamientos singulares (estatales) se determinan solamente con una capacidad de autodeterminación limitada a los acuerdos bilaterales o a la normativa global.

Cuando se discute la globalización y el Derecho administrativo hay que tomar conciencia que el Derecho por lo general opera como normatividad de un Estado nacional. Si bien los Estados son considerados 
soberanos, los procesos reales de "globalización" reducen cada vez más su capacidad de decidir autónomamente sobre sus legislaciones fundamentales. Existe una organización transnacional que impone sus reglas a los ordenamientos jurídicos domésticos en función a lo que en el seno de estas organizaciones se considera la solución ideal, lo cual — evidentemente - no responde a intereses nacionales, sino más bien a los intereses de estas organizaciones diversas ${ }^{6}$.

De esta manera, es indiscutible que los intereses foráneos de los Estados Unidos, a través de los TLC, determinan nuestras normas nacionales por sobre los intereses públicos "soberanos". Estas normas, entonces, se ven modificadas a fin de amoldarse a aquellas impuestas por el TLC, tal como se hizo en la actualidad con los mencionados 99 decretos legislativos.

Dicho en otras palabras, los que antes aparecían como tratados bilaterales o multilaterales entre Estados soberanos se han convertido en expresiones de procesos que, de facto, ponen en tela de juicio la soberanía de los Estados, y —algunas veces_ la misma existencia de ellos?

Este caso es bastante ilustrativo, ya que arroja una luz sobre las posibilidades de un Estado como el peruano en el ámbito global. Visiblemente, un país como el Perú tiende a perder su soberanía y autonomía en la normatividad - incluso dentro de su territorio- debido a actores externos que, por presión, imposición o negociación, logran imponer una normatividad que coincida con sus intereses - tal es el caso del TLC con los Estados Unidos-.

Por otro lado, es muy difícil establecer un sistema de control de cumplimiento de las disposiciones de los tratados o de los convenios

6 Los sistemas legales de protección de los derechos de la propiedad intelectual, universalizados a través de los convenios internacionales o de organizaciones como la Organización Mundial de la Propiedad Intelectual (OMPI) o la Organización Mundial del Comercio (OMC), no necesariamente benefician a los países en desarrollo cuyo producto bruto interno (PBI) no depende de los ingresos provenientes de los derechos de propiedad intelectual (DPI).

7 Algunos estados, como los de la Unión Europea (UE), han cedido parte de su soberanía a nuevas instancias supranacionales. En el citado caso de la Unión Europea, estas cesiones de la soberanía aparecen como acuerdos tomados entre los estados con el fin de crear una normatividad generalizada bajo objetivos comunes. En la UE esto es coherente, ya que sus miembros en realidad tienen economías tan entrelazadas — con procesos de producción cuyas partes se realizan en estados diversos- que una normatividad es imperativa para facilitar el funcionamiento económico, el traslado de personas, la cooperación en programas de investigación, el reconocimiento generalizado de educación superior, etcétera. En realidad, la UE es el caso de una globalización administrativa coordinada y equitativa entre muchos estados, a fin de que no solo se puedan crear las bases de su funcionamiento interior, sino también su presencia global como bloque. En cierto grado, parecería ser la abdicación de los estados nacionales y la creación de una nueva forma de normatividad supranacional. Este caso es ilustrativo, debido a que la Unión Europea ha creado mecanismos que permiten que el desarrollo de los estados integrados por ella resulte más o menos parejo por ejemplo, el traslado de fondos para estados con un desarrollo anterior no competitivo con los otros miembros del bloque-. Asimismo, ha creado una normatividad administrativa económica que asegura que el mercado integrado en la manufactura de productos no pueda ser monopolizado por una empresa o por empresas que operen desde otros ámbitos globales considerados lesivos a los intereses comunes. 
internacionales si es que no se tiene un esquema de accountability. Por ello, el debate se concentra actualmente en qué sistema de rendición de cuentas o de responsabilidad podría aplicarse a nivel internacional o supraestatal, habida cuenta de que los conceptos del Derecho administrativo pensados para un Estado soberano sirven $-\mathrm{y}$ no en poco - de base para el diseño de un suprasistema, y que esta -al mismo tiempo- es una de las partes de lo que se llama "Derecho administrativo global"s.

En efecto, la proliferación de la regulación transgubernamental de alcance mundial o regional en diversas materias — regulación económica, banca, ambiente, derechos humanos, biodiversidad, pesquería, laboral, propiedad intelectual, etcétera- marca el nacimiento de lo que se propone denominar un Derecho administrativo global, el cual trata de explicar cuáles son las reglas en los procesos de toma de decisiones de estas organizaciones, así como también en las relaciones entre los Estados, las organizaciones no gubernamentales, los órganos reguladores u órganos administrativos encargados de aplicar los convenios, los tribunales administrativos internacionales, los derechos de participación de la sociedad, la obligatoriedad de la regulación internacional para los Estados nacionales que son parte, para su influencia en las decisiones domésticas de los diversos países, o para la creación de mecanismos de accountability que permitan rastrear si aquellas normas se están cumpliendo o no, como ocurre hoy en día.

Al Perú no le queda otro camino - y no es un camino incorrecto- que el de tratar de adherirse a acuerdos multilaterales que de alguna forma presenten un marco jurídico supraestatal, con instancias de arbitraje entre los Estados que los han suscrito. Claro está que estos acuerdos multilaterales algunas veces no velan por los intereses generales de los firmantes, sino por el de los Estados que han promovido su creación en el marco de la ONU o a partir de conferencias multilaterales.

Este enjambre jurídico global es la manifestación de los cambios históricos producidos en el mundo desde la globalización y se relaciona directamente con el objeto de estudio del presente trabajo: la globalización jurídica y los límites de los poderes públicos en el caso de las contrataciones estatales del Perú.

Como mencionamos al inicio, la globalización se extiende sin duda al ámbito jurídico. Cada vez es más frecuente que el análisis legal de temas domésticos necesariamente involucre una consulta a las normas que

8 De nada serviría un sistema global que pretenda pautar las soluciones a los problemas causados por el cambio climático si todos los países no ratifican el convenio —como Estados Unidos, que no ha ratificado el Protocolo de Kioto-, si los países que son parte no demuestran que han cumplido con introducir y aplicar los mecanismos propuestos por las normas, o si las decisiones tomadas en un asunto de importancia mundial no han tenido en cuenta las opiniones y el sentir de la humanidad organizada con asociaciones privadas (ONG y otros).

\section{ACUERDOS DE PROMOCIÓN \\ COMERCIALY \\ CONTRATACIONES ESTATALES:}

ESPECIAL

REFERENCIA A LOS

LÍMITES DE LOS

PODERES PÚBLICOS

EN EL ESPACIO

JURÍDICO GLOBAL

COMMERCIAL

MARKETING

AGREEMENTS AND STATES CONTRACTS 
conforman el ordenamiento jurídico global en las materias más diversas, como la "educación, ciencia y cultura, agricultura y alimentación, sanidad, trabajo, medio ambiente, ayuda al desarrollo, cooperación monetaria y financiera, comercio internacional, propiedad intelectual, transportes terrestres, espacios marítimos, especio, comunicaciones, energía nuclear, etcétera"

Y en vista de que el Derecho administrativo es vasto y global, sus instituciones, en efecto, están influenciadas por el sistema jurídico global. Tal es el caso del medio ambiente, de la biodiversidad, del acceso a los recursos géticos, de los procedimientos administrativos de registro de patentes, de los medicamentos y de la salud, y también, en lo que es objeto del presente estudio, de las contrataciones estatales.

En esta ocasión, la ponencia explicará la manera en que el capítulo 9 del Tratado de Libre Comercio entre Perú y Estados Unidos, titulado "Contratación pública del TLC", ha limitado el poder normativo del Estado peruano.

\section{CONTRATACIONES ESTATALES PERUANAS Y EL TLC PERÚ-ESTADOS UNIDOS DE AMÉRICA}

\section{II.1. Marco general}

Para cumplir las funciones públicas o las actividades que les han sido asignadas por la Constitución y las leyes, las entidades de la administración pública y las empresas públicas pertenecientes a la actividad empresarial del Estado requieren aprovisionarse de los bienes, servicios y obras necesarios para ello. Las contrataciones estatales tienen por finalidad satisfacer esta exigencia de provisión de lo necesario.

Como es lógico, cuando la administración pública necesita comprar bienes, adquirir servicios o contratar empresas que construyan una obra pública con los recursos públicos de sus presupuestos, está obligada a hacerlo de una manera transparente y pública, a fin de elegir la propuesta más adecuada para satisfacer el interés público que subyace a toda contratación del Estado.

El cauce legal para hacerlo es el de los procesos de selección o procesos licitatorios en sus diversas modalidades (licitación pública, concurso público, adjudicación directa pública, adjudicación directa selectiva y adjudicación de menor cuantía), dependiendo del objeto y del monto de contratación. Estos procesos constituyen mecanismos para la formación de la voluntad de la administración pública y el perfeccionamiento del contrato correspondiente. 
Al respecto, la Constitución Política de 1993 así lo establece en su artículo 76:

Artículo $76^{\circ}$ - Las obras y la adquisición de suministros con utilización de fondos o recursos públicos se ejecutan obligatoriamente por contrata y licitación pública, así como también la adquisición o la enajenación de bienes. La contratación de servicios y proyectos cuya importancia y cuyo monto señala la Ley de Presupuesto se hace por concurso público. La ley establece el procedimiento, las excepciones y las respectivas responsabilidades" (el subrayado es nuestro).

Sobre el particular, el Tribunal Constitucional, en la sentencia recaída en el expediente número 020-2003-AI/TC, deja sentado en su fundamento 12 que:

La función constitucional de esta disposición es determinar y, a su vez, garantizar que las contrataciones estatales se realicen necesariamente mediante un procedimiento peculiar que asegure que los bienes, servicios u obras se obtengan de manera oportuna, con la mejor oferta económica y técnica, y respetando principios tales como la transparencia en las operaciones, la imparcialidad, la libre competencia y el trato justo e igualitario a los potenciales proveedores. En conclusión, su objeto es lograr el mayor grado de eficiencia en las adquisiciones o enajenaciones efectuadas por el Estado, sustentado en el activo rol de los principios antes señalados para evitar la corrupción y malversación de fondos públicos [...] (el subrayado es nuestro).

De lo expuesto, queda claro que la Constitución establece como regla general el que se realice un proceso de selección para las contrataciones estatales de servicios y, a la vez, delega a la ley de la materia —en este caso, la Ley de Contrataciones del Estado- que establezca las reglas de las licitaciones públicas y de los otros procesos de selección, así como las excepciones a la realización de los procesos de selección. Nótese que hay una doble lógica en la que se funda el proceso de selección: el eficiente y transparente uso de los fondos públicos, por un lado, y la satisfacción del interés público, por el otro.

En palabras del profesor Comadira, "la licitación pública es un modo de selección de los contratistas de entes públicos en ejercicio de la función administrativa, por medio del cual éstos invitan, públicamente, a los posibles interesados para que, con arreglo a los pliegos de bases y condiciones pertinentes, formulen propuestas de entre las cuales se seleccionará la más conveniente al interés público"10.

ACUERDOS DE PROMOCIÓN

COMERCIALY

CONTRATACIONES

ESTATALES:

ESPECIAL

REFERENCIA A LOS

LÍMITES DE LOS

PODERES PÚBLICOS

EN EL ESPACIO

JURÍDICO GLOBAL

COMMERCIAL

MARKETING

AGREEMENTS AND STATES CONTRACTS 
Ciertamente, la doctrina administrativa es unánime en afirmar que:

La elección para que la licitación opera como cauce formal es de fundamental importancia porque si el fin último que persigue la Administración es asegurar con inmediatez el bien común en tanto causa final del Estado, el desenvolvimiento de la actividad de selección y su concreción suponen, por un lado, el cumplimiento de los condicionamientos jurídicos formales del procedimiento y, por otro, su conclusión con un acto justo y eficaz ${ }^{11}$.

A esto hay que agregar que todos los documentos y las etapas del proceso deben responder a los principios de transparencia, publicidad y libre competencia consagrados en la Ley de Contrataciones y Adquisiciones del Estado.

Sin embargo, este poder de autodeterminación de la normativa, aplicable a los procesos de selección, está limitado a las reglas establecidas de mutuo acuerdo en el TLC entre Perú y Estados Unidos de América. Esto en varios aspectos en los que el TLC determina el contenido de la legislación nacional, como se verá a continuación.

\section{II.2. Capítulo 9 del TLC}

\section{II.2.1. Ámbito de aplicación}

El capítulo 9 del TLC se refiere a las contrataciones públicas de mercancías, servicios o ambos; a las de compra, alquiler con o sin opción de compra; y a las de construcción, operación, transferencia, concesión de obras públicas, productos digitales, etcétera. Estas compras solo estarán en condición de "cubiertas" por el TLC dependiendo de un aspecto subjetivo referido a las entidades o empresas incluidas; o de un aspecto objetivo, de acuerdo al tipo de contrato y a sus montos:

Capítulo 9 del TLC

Ámbito de aplicación

Contratación pública de mercancías, servicios o ambos cubiertas, según:

a. Tipo y objeto de contrato

b. Valor de la contratación

c. Entidad contratante [...] (el subrayado es nuestro).

\section{II.2.1.1. Tipo y objeto del contrato}

Son considerados los contratos de compra, alquiler o arrendamiento, con o sin opción de compra, construcción-operación-transferencia y contratos de concesión de obras públicas. 


\section{II.2.1.2. Valor de la contratación}

Los valores de los bienes o servicios a adquirir son un factor indispensable para determinar qué contratación se encuentra comprendida en el capítulo 9 del TLC, y son expresamente fijados a través de umbrales para el "nivel central de gobierno" (gobierno nacional), el "nivel sub central de gobierno" (gobiernos regionales y locales) y otras entidades cubiertas, básicamente las empresas estatales.

\section{Valor - Umbrales anexo 9.1}

Gobierno nacional

Sección A: Nivel Central de Gobierno para compras mayores
o igual a:

Gobiernos Regionales y Gobiernos Locales

ACUERDOS DE

PROMOCIÓN

COMERCIALY

CONTRATACIONES

ESTATALES:

ESPECIAL

REFERENCIA A LOS

LÍMITES DE LOS

PODERES PÚBLICOS

EN EL ESPACIO

JURÍDICO GLOBAL

COMMERCIAL

MARKETING

AGREEMENTS AND

\begin{tabular}{|c|}
\hline $\begin{array}{l}\text { Sección B: Nivel Sub Central de Gobierno para compras } \\
\text { mayores o igual a: }\end{array}$ \\
\hline Mercancías y servicios: $\quad$ U.S. \$ 526000.00 \\
\hline Servicios de construcción: U.S. \$ 7407000.00 \\
\hline Tiene notas especiales \\
\hline Empresas estatales y otras entidades cubiertas \\
\hline $\begin{array}{l}\text { Sección C: Otras entidades cubiertas para compras mayores } \\
\qquad \text { o igual a: }\end{array}$ \\
\hline Lista A: empresas de la actividad empresarial del Estado (23) \\
\hline Mercancías y servicios: $\quad$ U.S. \$ 250000.00 \\
\hline Servicios de construcción: U.S. \$ 7407000.00 \\
\hline Tiene notas especiales \\
\hline
\end{tabular}




\subsubsection{Elemento subjetivo}

No se aplica el TLC a más de 2 mil instituciones públicas, ya que este solo resulta de aplicación a las entidades que se encuentran en los listados que, como anexo, forman parte de aquel instrumento internacional.

Los organismos públicos incluidos en las listas pertenecen al gobierno nacional, a los gobiernos regionales y locales, e involucra también a organismos constitucionales autónomos, así como organismos de empresas estatales. Del mismo modo, exceptúa de su aplicación a ciertas entidades para contrataciones determinadas.

En conclusión, para saber qué entidades están sujetas al TLC, es necesario revisar sus anexos y combinar este aspecto subjetivo con el tipo de contrato y los umbrales antes mencionados. Cuando confluyan estos tres elementos (entidad, contrato y valor) estaremos frente a una contratación cubierta por el TLC. Y esto quiere decir sujeta a sus disposiciones.

Para las contrataciones cubiertas, la regla del TLC — coincidente con la Constitución Política de 1993- es que se dé un procedimiento de licitación pública, el cual se entiende como el proceso público de selección que observa las reglas que pormenorizadamente establece el TLC.

Si bien es cierto que existe obligación de hacer un proceso licitatorio para las compras, como en todo sistema de contratación también el TLC establece excepciones al proceso de selección "general", y estos son los casos en los cuales son aplicables los numerales 9.7.3, 9.7.4, 9.7.5 (por invitación, listas multiusos) y 9.8 (contratación directa) del capítulo 9 del TLC. Si hacemos un paralelo con nuestra legislación nacional, se podría decir que existen procedimientos con convocatoria pública y abierta y otros por invitación, además de las exoneraciones que permiten una contratación directa.

\section{II.2.2. Obligaciones para las contrataciones estatales derivadas del TLC}

Si estamos frente a una contratación cubierta por el TLC y existe obligación de llevar a cabo un proceso licitatorio abierto, la normativa nacional —según el TLC_ - debe considerar indispensables, como mínimo, los siguientes aspectos:

a. Publicación de los planes anuales de contrataciones.

b. Avisos de contratación futura (convocatoria o invitación): publicación SEACE o periódica.

c. Plazo para la presentación de propuestas:

- El plazo es de un mínimo cuarenta (40) días contados desde la fecha de publicación del aviso de contratación futura o, en el caso de licitación selectiva, desde la fecha en que la entidad invita a los postores a participar. 
- Este plazo puede reducirse a treinta (30) días y nunca menos de diez (10) días siempre que: (i) la entidad haya publicado un aviso de contratación futura (convocatoria) en un medio electrónico y simultáneamente haya provisto de los documentos de contratación; (ii) sea suficiente para que el postor presente una oferta; y (iii) solamente en los casos señalados en el numeral 4 del artículo 9.5 del capítulo 9.

d. Documentos de contratación, es decir, toda la información necesaria para preparar y presentar ofertas, entre las que se encuentran la naturaleza de la contratación, las especificaciones técnicas la cantidad, las garantías, los criterios, el valor del precio, el calendario del proceso (por ejemplo, fecha para la presentación de propuestas). En cuanto a las especificaciones técnicas, el texto establece expresamente reglas como la prohibición de colocar marcas o nombre comercial, patente, derecho de autor, entre otros.

e. Fecha y reglas para la recepción y apertura de ofertas.

f. Publicación de la información sobre la adjudicación.

g. Posibilidad de impugnación de proveedores ante un órgano independiente e imparcial.

h. Ambas partes tienen la obligación de informar sobre la normativa y precedentes.

A partir de lo expuesto, no habría diferencia sustancial con nuestra legislación. Esto se debe a que la Ley de Contrataciones del Estado (decreto legislativo 1017) fue aprobado por el Poder Ejecutivo por expresa delegación del Congreso de la República al implementar el TLC en nuestro país. Sin embargo, hay algunas notas especiales, de las cuales resaltaremos solo dos.

\section{II.2.2.1. Requisitos de participación para ser postor}

Respecto a las condiciones de participación establecida por el artículo 9.7 del capítulo 9, el TLC establece que:

Artículo 9.7: Condiciones de Participación. Requisitos generales

Cuando una entidad contratante solicite que los proveedores deban satisfacer ciertas condiciones de participación, la entidad deberá, sujeta a las demás disposiciones de este Capítulo: [...]

c) no impondrá como condición de participación en una contratación pública, que a un proveedor se le hubiese sido adjudicado uno o más contratos por una entidad contratante de la Parte de la entidad contratante o que el proveedor posea experiencia previa de trabajo en el territorio de la Parte [...]. 
El artículo glosado establece que no podría exigirse como un requisito para ser postor que este haya suscrito un contrato con una entidad pública de nuestro país o que posea experiencia en el Perú. Ello, se entiende, para evitar que las empresas de los Estados Unidos de América que no hayan desarrollado anteriormente en el país actividades puedan sean excluidas de las contrataciones estatales a través de la exigencia de este requisito. De esta manera, en la formulación de las bases, los organismos públicos de un proceso de selección —que tenga un objeto de contratación en el que puedan participar empresas estadounidensesno podrán exigir experiencia previa en el país. Esta es una muestra de lo afirmado anteriormente en el presente trabajo, en el sentido de que el TLC determina las reglas de contratación y la práctica administrativa.

\subsubsection{Impugnaciones por parte de empresas estadounidenses en los procesos de selección}

El artículo 9.11 (Revisión Nacional de las Impugnaciones de los Proveedores) del TLC dispone que cada parte debe garantizar que los proveedores puedan apelar la decisión inicial ante un órgano administrativo o judicial imparcial, un órgano que sea independiente de la entidad contratante o del objeto de la impugnación.

En este sentido, existe un sistema dual de impugnaciones en las contrataciones: por un lado, existe un sistema general para las empresas peruanas y extranjeras (no estadounidenses), las cuales impugnarán ante la misma entidad; por otro, se tiene un sistema especial, aplicable solo a las empresas estadounidenses.

Las normas peruanas lo disponen de la siguiente manera:

Decreto Legislativo № 1017, Ley de Contrataciones del Estado

Décimo Tercera.- Para definir la instancia que resolverá los recursos impugnativos que se interpongan en los procesos de selección en los que participen proveedores que provengan de países con los que la República del Perú tuviera vigente un tratado o compromiso internacional que incluya disposiciones sobre contrataciones públicas, se aplicarán, de ser el caso, los criterios establecidos en las mismas.

Decreto Supremo № 184-2008-EF

Artículo 104.- Recurso de apelación

Mediante recurso de apelación se impugnan los actos dictados durante el desarrollo del proceso de selección, desde la convocatoria hasta aquellos emitidos antes de la celebración del contrato. [...]

En aplicación de la Décimo Tercera Disposición Complementaria y Final de la Ley, el Tribunal será competente para conocer y resolver las controversias que surjan en los procesos de selección de las contrataciones que se encuentren bajo los alcances de tratados o 
acuerdos internacionales donde se asuman compromisos en materia de contratación pública.

De lo expuesto, queda meridianamente claro cómo un TLC condiciona la autodeterminación normativa doméstica, la cual tiene que adecuarse al TLC para no contravenirlo.

\section{II.2.3. Excepciones a la licitación pública}

La regla de la licitación pública tiene excepciones a través de un proceso por invitación denominado "licitación selectiva", el cual correspondería a lo que en la legislación peruana se denomina "adjudicación directa selectiva" y "adjudicación de menor cuantía".

El sistema de compras mediante listas multiusos es otra modalidad permitida por el TLC. Ella se refiere a que la entidad puede contar con lista de proveedores para determinados servicios y bienes; ella está obligada a invitar a las empresas de la lista a todas las licitaciones que se realicen para dichos bienes y servicios. Para utilizar este mecanismo, cada entidad debe cumplir las siguientes condiciones:

a. describir los bienes y servicios sujetos a esta modalidad;

a. verificar las condiciones de participación de los postores y los mecanismos para comprobar la veracidad de aquellas;

b. probar los datos de la entidad;

c. cumplir los plazos de la presentación de solicitudes para integrar las listas multiuso; $\mathrm{y}$

d. emplear las listas multiuso de acuerdo con el capítulo 9 del TLC.

\section{II.2.3.1. Contratación directa (9.8 del capítulo 9)}

La contratación directa por causales de excepción — lo que se denomina "exoneraciones" en la legislación peruana- presupone que en caso alguno se busque impedir la competencia, entendiéndose por esto que no se vea la forma de proteger a los proveedores nacionales o de discriminar a las empresas de la otra parte.

El referido artículo 9.8 permite realizar contratación directa en siete supuestos, lo cual posibilita no aplicar los artículos 9.4, 9.5, 9.6, 9.7, 9.9.1, 9.9.3 9.9.4 9.9.5, 9.9.6 y 9.9.7-del TLC (ver infra). Cabe señalar que las entidades públicas de las partes pueden contratar a un proveedor o a proveedores de su elección, así como pueden considerar la no aplicación de los citados artículos, los que se explicitan resumidamente en la tabla siguiente. 


\section{Tabla: Contratación directa}

9.4 Avisos de contratación futura

9.5 Plazos para presentación de ofertas

9.6 Información sobre contrataciones públicas futuras

9.7 Condiciones de participación

9.9 Recepción y apertura de ofertas

9.9.3 Recepción y apertura de ofertas

9.9.4 Adjudicación de contratos

9.9.5 Adjudicación de contratos

9.9.6 Adjudicación de contratos

9.9.7 Adjudicación de contratos
Avisos de contratación futura (convocatoria o invitación) publicación SEACE o periódico. Publicación de los Planes Anuales de Contrataciones

40 días para presentación de propuestas, reducible a 30 días o menos. Nunca menos de 10 días

Proporcionarla oportunamente, cuando sea solicitada, documentos de contratación con toda la información necesaria (bases)

Criterios objetivos para valorar: capacidad legal, comercial, técnica, financiera, incluyendo actividades en nuestro territorio y en el de la otra parte. Prohibido exigir contrato anterior en el Perú o que posea experiencia en el país. Lista Multi-Usos de proveedores

Apertura de sobres garantía de igualdad e imparcialidad.

Dar la oportunidad a todos los proveedores de corregir errores involuntarios

Presentar oferta por escrito y el día de la apertura de sobres. Cumplir requisitos esenciales y criterios de evaluación de las bases

No otorgar la buena pro al ganador si atenta contra el interés público

Impedimento de cancelar la contratación pública, ni terminar o modificar un contrato con el fin de evadir el Cap. 9

Comunicar a los proveedores quien ganó la buena pro y, a solicitud, dar a los perdedores las razones por las que no ganaron

Los siete supuestos de excepción a la licitación pública o a la licitación selectiva - aquello que es conocido en nuestra legislación como "causales de exoneraciones" o "contrataciones adicionales o complementarias" - son los siguientes:

\section{Primer supuesto}

Si hubo una convocatoria o invitación realizada con anterioridad y sucedió lo siguiente:

- no se presentó oferta alguna;

- ninguna oferta cumplió los requisitos esenciales; o

- ningún proveedor cumplió con las condiciones de participación. 
Es el caso del proveedor único o el bien o servicio que no admite sustituto debido a que:

- el objeto es la compra de una obra de arte;

- se protegen los patentes del autor o de otros derechos exclusivos; $\mathrm{O}$

- existe ausencia de competencias por razones técnicas.

\section{Tercer supuesto}

No requieren de proceso de licitación pública los adicionales a los contratos de mercancías y servicios.

\section{Cuarto supuesto}

Considerado para mercancías adquiridas en el mercado de productos básicos.

\section{Quinto supuesto}

Adquisición de un prototipo o de una primera mercancía o servicio que es desarrollado a solicitud de la propia entidad.

\section{Sexto supuesto}

En la medida en que sea estrictamente necesario: cuando, por ejemplo, por razones de extrema urgencia ocasionadas por acontecimientos imprevistos por parte de la entidad contratante, no se pueda obtener las mercancías o servicios a tiempo y mediante procedimientos consistentes con los artículos del 9.4 hasta el 9.7. Asimismo, cuando el uso de esos procedimientos podría ocasionar un grave perjuicio para la entidad contratante o la parte correspondiente.

Esta causal es semejante a la de emergencia contemplada por nuestra legislación ${ }^{12}$.

\section{Séptimo supuesto}

Excepción de los adicionales de obra: esto se aplica solo en caso de circunstancias imprevistas y cuando aquellos resulten necesarios para

\footnotetext{
12 Decreto legislativo 1017, Ley de Contrataciones del Estado: "Artículo 23.- Situación de emergencia. Se entiende como situación de emergencia aquella en la cual la Entidad tenga que actuar de manera inmediata a causa de acontecimientos catastróficos, de situaciones que supongan grave peligro, o que afecten la defensa y seguridad nacional. En este caso, la Entidad queda exonerada de la tramitación del expediente administrativo y podrá ordenar la ejecución de lo estrictamente necesario para remediar el evento producido y satisfacer la necesidad sobrevenida, sin sujetarse a los requisitos formales del presente Decreto Legislativo. El Reglamento establecerá los mecanismos y plazos para la regularización del procedimiento correspondiente. Las demás actividades necesarias para completar el objetivo propuesto por la Entidad no tendrán el carácter de emergencia y se contratarán de acuerdo a lo establecido en la presente norma".
}

\section{ACUERDOS DE PROMOCIÓN COMERCIALY \\ CONTRATACIONES ESTATALES: \\ ESPECIAL \\ REFERENCIA A LOS \\ LÍMITES DE LOS \\ PODERES PÚBLICOS \\ EN EL ESPACIO \\ JURÍDICO GLOBAL \\ COMMERCIAL \\ MARKETING \\ AGREEMENTS AND STATES CONTRACTS}


completar el servicio de construcción del contrato. No excederá el 50\% del contrato original.

Toda excepción debe ser informada a solicitud de la otra parte. La entidad que contrató directamente informará:

a. el nombre de la entidad;

a. el valor y la descripción del objeto del contrato; y

b. la declaración, indicando las circunstancias y condiciones que justificaron el procedimiento distinto a la licitación pública o, lo que es lo mismo, explicando la licitación selectiva y la causal utilizada.

\section{REFLEXIÓN FINAL}

Luego del análisis del presente trabajo, queda claro que las reglas del TLC imponen las normas en el ordenamiento nacional. El punto a resaltar es que el poder de autodeterminación normativa del país se ve relativizado por el mencionado acuerdo o por cualquier otro TLC o acuerdo internacional celebrado sobre la materia. Esta situación es la expresión de la globalización jurídica, así como de una de sus características: la relativización de la soberanía nacional.

Cualquier cambio en la legislación nacional — tales como la emisión de decretos de urgencia para establecer contrataciones directas o como los procesos especiales de selección para proyectos de inversión priorizados - solo podrá ser emitido siempre que se enmarque en el TLC. En caso contrario, se expondrían a un incumplimiento del contrato, y las consecuencias de ello las sufriría el país.

Como cuestión final, debemos mencionar que para las contrataciones estatales no solo debe tenerse en cuenta la Ley de Contrataciones del Estado y su reglamento, sino este TLC entre Perú y Estados Unidos de América y los demás tratados que se aprueben e incluyan un capítulo sobre la materia. Además, claro, de los diversos decretos de urgencia y las leyes que a lo largo de estos cinco años han expedido el gobierno y el congreso, respectivamente. Todo esto representa una verdadera complicación para el operador jurídico, tanto del lado de la administración pública como para el del empresariado, dado que existen alrededor de cinco regímenes paralelos creados por ley o decretos de urgencia que, en lugar de simplificar y transparentar el sistema, lo tornan extremadamente complejo. 